\title{
ELEMENTOS FENOMENOLÓGICOS PARA UNA FILOSOFÍA DEL DEPORTE. EL CUERPO PROPIO Y LAS HABILIDADES EN EL CASO DEL TRIATLÓN ${ }^{1}$
}

\author{
PHENOMENOLOGICAL ELEMENTS FOR A PHILOSOPHY OF SPORTS: \\ BODY AND SKILLS IN THE CASE OF THE TRIATHLON
}

ELEMENTOS FENOMENOLÓGICOS PARA UMA FILOSOFIA DO ESPORTE.
O CORPO PRÓPRIO E AS HABILIDADES NO CASO DO TRIATLO

Alejandro R. Laregina ${ }^{2}$

Resumen

El triatlón es un deporte de pruebas combinadas que reúne la natación, el ciclismo y el pedestrismo, y presenta una gran complejidad tanto en aspectos relativos a la dimensión fisiológica, psicológica, como también en la fenomenológica. En este artículo me propongo llevar a cabo una presentación esquemática de la estructura del triatlón, y realizar una descripción fenomenológica de aspectos relativos al cuerpo propio, a la orientación vivida y habitualidades presentes en esa estructura para luego llevar a cabo una reflexión a partir de la misma. La relevancia filosófica de esta tarea radica en que podría aportar herramientas conceptuales para la clarificación de la experiencia vivida por el triatleta, la cual muchas veces queda imposibilitada de ser expresada y transmitida. A su vez, podría mostrarse el alcance y la adecuación de los conceptos utilizados en la descripción de la práctica del triatlón como fenómeno del mundo de la vida deportiva.

Palabras clave: natación; ciclismo; pedestrismo; ritmo; esquema corporal

\begin{abstract}
A triathlon is a sport of combined events that includes swimming, cycling and running, and which is very complex in terms of the physiological, psychological and phenomenological dimension. In this paper, I propose doing a schematic presentation of the structure of the triathlon and a phenomenological description of aspects related to one's own body, to the orientation lived, and to habitualities present in that structure, followed by a reflection based on it. The philosophical relevance of this task is that it could provide conceptual tools to clarify the experience lived by the triathlete, which is often impossible to express and to transmit. It could also show the scope and adequacy of the concepts used to describe the practice of the triathlon as a phenomenon of the world of sport life.
\end{abstract}

Keywords: swimming; cycling; running; rhythm; body schema

1 Este artículo se escribió en Argentina, en el marco de los proyectos de investigación (2014-2017), PICT 2014-2137. Agencia Nacional de Promoción Científica y Tecnológica (ANPCyT). Título: "Proyecto de reactualización contemporánea de la fenomenología: fenomenología de la corporeidad"; Responsable: Dra. Ariela Battán Horenstein; y (2014-2017) UBACYT 20020130100041BA. Título: "Fenomenología, hermenéutica y lógica trascendental. Los niveles fenomenológicos, protofenomenológicos e intrahermenéuticos del análisis trascendental. Los niveles paratrascendental y no trascendental del análisis fenomenológico y hermenéutico". Director: Dr. Roberto Walton; codirector: Dr. Horacio Mario Ramón Banega.

2 Profesor en Enseñanza Media y Superior en Filosofía, docente Departamento de Filosofía, Universidad de Buenos Aires. 


\section{Resumo}

O triatlo é um esporte composto por uma combinação de provas de natação, ciclismo e corrida, e apresenta uma grande complexidade tanto em aspectos relacionados à dimensão fisiológica e psicológica quanto à fenomenológica. Neste artigo visamos realizar uma apresentação esquemática da estrutura do triatlo e realizar uma descrição fenomenológica dos aspectos relacionados com o corpo, a orientação vivenciada e duração e habitualidades presentes nessa estrutura, para em seguida realizar uma reflexão a partir desta. A relevância filosófica desta tarefa é que poderia fornecer ferramentas conceituais para o esclarecimento da experiência experimentada pelo triatleta, que muitas vezes é impossível de ser expressa e transmitida. Por sua vez, poderia mostrar o escopo e a adequação dos conceitos utilizados na descrição da prática do triatlo como um fenômeno do mundo dos esportes.

Palavras-chave: natação; ciclismo; corrida; ritmo; esquema corporal

Fecha de recepción: 16 de agosto de 2017

Fecha de aprobación: 9 de julio de 2018

Para citar este artículo:

Laregina, A. (2017). Elementos fenomenológicos para una filosofía del deporte. El cuerpo propio y las habilidades en el caso del triatlón. Lúdica Pedagógica, 26, 23-32. 
INTRODUCCIÓN: FILOSOFÍA DEL DEPORTE Y FENOMENOLOGÍA

La filosofía del deporte es una disciplina que tiene un surgimiento relativamente reciente atravesada por múltiples dimensiones: éticas, gnoseológicas, metafísicas y estéticas. En todas estas dimensiones, la reflexión filosófica sobre el cuerpo tiene un lugar destacado (Pérez, 2011, pp. 73-74). Se puede observar la presencia de la corporalidad como tópico desde el primer número del Journal of Philosophy of Sport publicación oficial de la International Association for the Philosophy of Sport-, editado en el año $1974^{3}$. Allí, aparecen avances sobre el tema que luego serán retomados por la corriente fenomenológica contemporánea. Por ejemplo, O’Neill en su artículo “The spectacle of the body" señala el retraso que tenemos en encontrar el cuerpo, pues su reconocimiento lo hacemos cuando este duele, tiene sobrepeso, hay una pierna fracturada ${ }^{4}$. Si bien el enfoque de $0^{\prime} \mathrm{Neill}$ no es estrictamente fenomenológico, en esa reflexión aparecen insinuados aspectos de la corporalidad que desarrollará profundamente Drew Leder en The absent body (1990), en relación con los grados de des-aparición del cuerpo.

La corriente fenomenológica cuenta con una breve reseña y revisión de bibliografía relevante para la filosofía del deporte realizada por Irina Martínková, en su artículo "Phenomenology and sport", en el Routledge Handbook of Philosophy of Sport. Martínková señala el texto de Slusher H., Man, Sport and Existence (1967), como un antecedente importante de la disciplina en el que se retoman autores, tales como Husserl, Heidegger, Sartre y Jaspers. Pero señala también que el enfoque estrictamente fenomenológico no tiene un fuerte impacto, sino hasta el siglo XXI ${ }^{5}$ (Martínková, 2015, pp. 180-181). En esta última etapa es retomado el pensamiento de Husserl y de Merleau-Ponty por distintos autores como Zahavi,

3 Como señala Pérez Triviño, se pueden encontrar reflexiones filosóficas relativas al cuerpo ya en los pensadores antiguos y modernos, no llegará el pensamiento sistemático del deporte desde un punto de vista filosófico, sino hasta el siglo xx (Pérez, 2011, pp. 73-74).

4 Cfr. O'Neill, quien llamativamente también incluye el amor y el embarazo como situaciones en que encontramos el cuerpo "demasiado tarde" (1974, p-110)

5 Señala específicamente Martínková, I. y Parry, J. (2012). Phenomenological approaches to sport, Nueva York: Routledge.
Gallagher y Dreyfus, entre otros. Algunos de los tópicos fenomenológicos que la autora señala como destacados para la reflexión filosófica del deporte son el aprendizaje de habilidades y la encarnación (embodiment). Estos tópicos nos remiten al pensamiento desplegado por Merleau-Ponty en Fenomenología de la percepción. En esta obra, el autor francés afirma que la conciencia no es originariamente un "yo pienso que", sino un "yo-puedo" (Merleau-Ponty, 1957, p. 149). El yo-puedo expresa la relación entre el cuerpo y el mundo, relación que presenta a la motricidad como elemento destacado. Merleau-Ponty afirma que "se aprende un movimiento cuando el cuerpo lo ha comprendido, esto es, cuando lo ha incorporado a su mundo" (1957, p. 151). Este aprendizaje del cuerpo no está expresado al modo del saber-qué, sino que se realiza en un saber-cómo. Este saber-cómo - sedimentado en el esquema corporal- solo puede ser ampliado a través de la práctica. Para dar cuenta de la captación motriz de una significación motriz se pueden presentar los ejemplos de aprender a andar en bicicleta o aprender a nadar.

El triatlón es un deporte de pruebas combinadas que reúne natación, ciclismo y pedestrismo, y presenta una gran complejidad tanto en aspectos relativos a la dimensión fisiológica, psicológica, como también en la fenomenológica.

Torres señala que el triatlón se divide en varias etapas: una primera etapa correspondiente a la natación, una segunda etapa correspondiente al ciclismo y una tercera etapa correspondiente a la carrera; y también las transiciones que nos llevan de la natación al ciclismo y del ciclismo a la carrera (2000, pp. 11-12). De esta manera, la estructura del triatlón contará con cinco momentos distintos que son pasibles de ser descritos a partir de conceptos fenomenológicos. El primer momento, la natación, tiene como característica destacada el envolvimiento de gran parte del cuerpo por el agua. La piel es tocada por el agua y el agua es tocada por la piel. La natación señala algunas características del cuerpo propio, tales como las sensaciones dobles, según son presentadas por Merleau-Ponty. A su vez, la orientación tal como es vivida pareciera sufrir cambios drásticos respecto al modo como es vivida en la cotidianeidad, pues mientras que en nuestra vida diaria, el arriba y el abajo, el adelante y el detrás, lo son en relación 
con nuestro cuerpo, en el fenómeno de la natación parecerían cambiar. El ciclismo presenta la inevitable relación del cuerpo con la bicicleta, aprendizaje de hábitos y síntesis del cuerpo propio con su espacialidad vivida y sus ejes de orientación una vez más cambiados, pues el desplazamiento consiste en un movimiento de rotación cíclica de las piernas que no tiene contacto con el piso. En tercer lugar, la carrera, donde se vuelven a cambiar los ejes de orientación vivida y el desplazamiento está a cargo de los pasos que realizan las piernas, pero con una vivencia luego de pedalear que genera una experiencia del cuerpo propio totalmente diferente a la que se podría tener al caminar o al correr como actividad única. A estos tres momentos se les suma la complejidad de las transiciones. El triatlón se presenta como un fenómeno complejo, pero pasible de una descripción fenomenológica. La relevancia filosófica de esta descripción fenomenológica radica en que podría aportar herramientas conceptuales para la clarificación de la experiencia vivida por el triatleta, experiencia que muchas veces queda imposibilitada de ser expresada y transmitida. A su vez, podría mostrarse la adecuación o no adecuación de los conceptos fenomenológicos a la descripción de la práctica del triatlón como fenómeno del mundo de la vida deportiva.

\section{ESTRUCTURA DEL TRIATLÓN: ETAPAS Y DESCRIPCIÓN FENOMENOLÓGICA}

En primer lugar, antes de ocuparnos de cada etapa del triatlón cabe realizar una breve descripción de las distintas modalidades y distancias del deporte. Como ya señalé previamente, el triatlón es un deporte de pruebas combinadas, que se realizan de forma continuada y siempre en el mismo orden: primero natación, luego ciclismo y por último pedestrismo. Se corre bajo distintas distancias y modalidades, la distancia olímpica, desde que forma parte de los Juegos Olímpicos de Sydney 2000, es 1500 m de natación, $40 \mathrm{~km}$ de ciclismo y $10 \mathrm{~km}$ de pedestrismo. Se corren también distancias más cortas en el triatlón sprint o short (750 m, $20 \mathrm{~km}, 5 \mathrm{~km})$ y en el super sprint ( $400 \mathrm{~m}, 10 \mathrm{~km}, 2,5 \mathrm{~km})$. Pero también se corren distancias mucho más largas, lo que la posiciona como la prueba de resistencia por excelencia en el deporte, por ejemplo, la distancia "ironman" (3800 m de natación, 180 km de ciclismo y, por último, una maratón de 42,195 km, para sumar 226 kilómetros totales). Es muy popular la distancia "half" o "medio ironman", que es justo la mitad de las distancias anteriores (1900 m, $90 \mathrm{~km}, 21 \mathrm{~km})$. Las pruebas de larga distancia se consideran de esfuerzo totalmente individual, por lo que no se permite la circulación de bicicletas en pelotón: un ciclista debe ir separado del otro por tres bicicletas o más según el reglamento de cada organización. A esta modalidad se le denomina "sin drafting", mientras que a la competencia olímpica se la considera "con drafting", y está permitido el rodamiento en pelotón. Por último, quería señalar que, en los Juegos Olímpicos de Río de Janeiro, hizo su debut la categoría de triatlón paraolímpico o paratriatlón, con distancia sprint. En todas las distancias y modalidades, se computa el tiempo final contabilizando tanto las tres etapas delimitadas de cada deporte así como también las dos transiciones.

\section{Primera etapa: la natación ${ }^{6}$}

En la primera etapa del triatlón, la natación, se pueden observar algunos aspectos del fenómeno del cuerpo en el agua que resultan fenomenológicamente relevantes en relación con el cuerpo propio: el predominio del sentido del tacto por sobre el resto de los sentidos, los cambios que se relacionan con el espacio vivido y la relación del cuerpo con el medio ambiente, $y$, por último, en este texto, me remitiré al aprendizaje de habilidades en el medio acuático y su relación con el esquema corporal. Estos puntos no agotan el tema, pero señalan la relevancia que puede tener la fenomenología para la filosofía del deporte, en especial para la natación y el triatlón. A su vez, el fenómeno de la natación y el cuerpo en el agua tienen relevancia para la fenomenología, ya que nos permite correr de nuestra experiencia cotidiana y así poner de relieve aspectos de nuestra experiencia vivida que nos pasan desapercibidas en la cotidianeidad.

En primer lugar, señalé como relevante la relación entre el sentido del tacto y el agua. En el triatlón existen distintos tipos de largadas, algunas desde tierra firme y otras ya con el cuerpo sumergido en el agua. En cualquier caso, los instantes previos a realizar la inmersión son escasos, y durante toda la etapa de natación, la cual consideraremos desde la largada

6 Respecto a este apartado en particular, cfr. Laregina (2013), en cuyos conceptos principales me he apoyado. 
hasta que el atleta se vuelva a poner de pie, el mismo se encuentra en contacto con el agua. Con-tacto, tacto, hace referencia a la relación de la piel con el agua. El sentido del tacto muestra una característica fenomenológica destacada en relación con el resto de los sentidos, las "sensaciones dobles" presentadas por Husserl, y luego, por Merleau-Ponty (1985, pp. 110-111). Podemos tocar, o ser tocados. Podemos tocar cognoscitivamente un objeto, activamente, explorando en este caso un medio, el agua, percibir su temperatura, su fluidez, su densidad. Pero también podemos ser tocados por el agua, en un "tacto pasivo", en zonas del cuerpo que parecen no tener esa capacidad exploratoria: el interior de las orejas, de la nariz, las axilas, el espacio entre los dedos, etc. Esos espacios corporales habitualmente se encuentran "desaparecidos" de la experiencia, no los notamos, no tomamos nota de ellos. Pero al ser tocados por el agua se vuelven presentes, cuando está todo nuestro cuerpo en el agua, somos tocados completamente, se nos ofrece un contorno de nuestro cuerpo propio, nos envuelve completamente el agua activando toda nuestra superficie táctil. Esta envoltura, esta contención, nos hace presente nuestro cuerpo propio, incluso presenta partes que el sentido de la vista no nos puede presentar, como ciertas partes de la espalda y la nuca. De esta manera, en la natación, aparece un cambio profundo en la experiencia de nuestro cuerpo vivido en relación con nuestra cotidianeidad, donde se encuentra desaparecido, como señalamos en la introducción, a menos que algo comience a fallar o doler. Esta preeminencia del sentido del tacto, que repercute en la constitución del cuerpo propio y el modo de vivenciar el propio cuerpo, también tiene relación con el modo en que habitamos el espacio.

El cuerpo propio es presentado por Merleau-Ponty como un sistema de acciones actuales o posibles, como vehículo de ser en el mundo, comprometido con un medio definido (1985, pp. 100-101). El filósofo francés hace una diferencia entre una espacialidad de posición, tal como la que tienen los objetos inanimados, de una espacialidad de situación, tal como la vivimos los seres humanos (p. 117). La situación viene a señalar que el cuerpo existe en relación con sus tareas y se encuentra polarizado hacia ellas. Esta situacionalidad manifiesta la centralidad del cuerpo propio en relación con el espacio y el resto de los objetos. Afirma Merleau-Ponty: "La palabra 'aquí, aplicada a mi cuerpo, no designa una posición determinada respecto a otras posiciones o con respecto a unas coordenadas exteriores, sino a la instalación de las primeras coordenadas [...]" (1985, p. 117). El arriba y el abajo, la derecha y la izquierda, así como lejano o cercano, siempre estarán anclados en el cuerpo propio. Es el cuerpo propio el que organiza el espacio vivido, y en el agua, así como cambia el modo de desplazamiento (ya no erguido sobre los pies), también cambia la orientación de los ejes vitales. Habitualmente, en nuestra vida cotidiana, el eje vital se encuentra en el plano horizontal (cfr. Merleau-Ponty, 2003, p. 24), nos movemos y nos desplazamos sobre el plano horizontal; en cambio, en la natación, el eje vital pareciera pasar a ser el vertical, el arriba y el abajo del nivel del agua se tornan determinantes. Poder respirar o no poder hacerlo (actividad del cuerpo orgánico y controlada, en mayor o menor medida por el cuerpo habitual) pareciera depender de tener las vías respiratorias por encima o por debajo del nivel del agua, del horizonte. A su vez, la información habitual respecto a la posición de los diversos objetos que se encuentran en nuestro medio, de la que disponemos gracias al sentido de la vista, en el agua se torna deficiente, debido al posicionamiento y cercanía del órgano visual respecto al nivel del agua. Sin embargo, frente a esta deficiencia respecto al conocimiento de nuestra posición en relación al resto de los objetos, el sentido del tacto nos profiere abundante información respecto a nuestra postura $^{7}$. Esta deficiencia se tornará relevante en el deporte de natación en aguas abiertas y en el triatlón, ya que el nadador debe reconocer la boya o las señales que proporciona el organizador de la carrera para seguir el circuito, realizando los trayectos de la manera más directa posible, lo que implica cierta coordinación entre postura y posición, para apuntar correctamente a esas boyas con el desplazamiento del propio cuerpo.

Este desplazamiento del propio cuerpo, en el medio acuático, toma distintos nombres, atendiendo a distintas variables. Cuando un cuerpo simplemente mantiene alguna parte suya por encima del nivel del agua, se dice que "flota", se mantiene a flote, esto

7 Para ver un profundo análisis del concepto de "postura" desde un punto de vista fenomenológico cfr. Serrano de Haro (2007, pp. 27-31). 
tanto para un barco de papel como para una pelota o un cuerpo. Si el cuerpo es un cuerpo vivo, se entiende que flota o está flotando si mantiene sus vías respiratorias por encima del nivel de agua (o flotación). Un objeto que flota, está a merced de la corriente de agua, no se está desplazando por cuenta propia. Si, en cambio, el cuerpo vivo se desplaza por cuenta propia, decimos que "nada" cuando lo hace respirando por encima de la superficie, o que "bucea" si lo hace por debajo. Ahora bien, estos tipos de movimientos se aprenden. Un movimiento se aprende cuando nuestro cuerpo lo comprende, cuando se consagra una intención motriz, esto es, cuando se experimenta una "concordancia entre aquello que intentamos y lo que viene dado, entre la intención y la efectuación" (Merleau-Ponty, 1985, p. 162). Así, decimos que sabemos nadar cuando hay concordancia entre nuestra intención de desplazarnos - cambiar de posición- en el medio acuático y aquello que logramos. Cada intención motriz consagrada ensancha nuestro arco intencional, nos hace capaces de realizar mayor cantidad de movimientos con sentido en el mundo. Cuanto más consagrado se halle un movimiento o una intencionalidad motriz, menos esfuerzo requerirá su ejecución. Por lo tanto, el entrenamiento desempeña un rol fundamental en este aspecto. La descripción fenomenológica puede continuarse con mayor o menor grado de detalle, de manera productiva, sin embargo, en este artículo, por cuestiones de espacio, dejaré solamente planteados estos tres aspectos (preeminencia del sentido del tacto, cambios en la orientación vivida y habilidades en el medio acuático) debido a su relevancia y dejaré abierta la descripción para ser continuada en futuras investigaciones; desde la utilización de distintos elementos, tales como antiparras, patas de rana, trajes de neopreno hasta la descripción de la sensibilidad al agua, característica determinante en el rendimiento de los nadadores y de raigambre profundamente fenomenológica.

\section{Primera transición: de la natación al ciclismo}

Una vez que el atleta se ha parado nuevamente en tierra firme, comienza la primera transición, que lo llevará desde el final de la natación hasta el parque cerrado en busca de su bicicleta y accesorios, tales como casco y zapatos de ciclismo. Es preciso aclarar que todo triatlón cuenta con una base llamada "parque cerrado" que delimita el espacio de los atletas, permitiéndoles ubicar su bicicleta y sus pertenencias antes del comienzo de la prueba. Antes de la largada, el atleta dispone su bicicleta, su casco y sus zapatos de ciclismo y de carrera de modo tal que puedan ser fácilmente accesibles para su uso, sin perturbar ni molestar a otros atletas. En la primera transición, el atleta pasa de nadar a correr hacia el parque cerrado en busca de su bicicleta y accesorios. Pasa de estar envuelto por el agua y tener el peso de su cuerpo en manos del medio acuático a ponerse de pie y cargar no solo con su peso sobre sus piernas, sino también con la fuerza de la gravedad. El cambio de eje se da violentamente, lo cual puede generar mareos y desorientación. Se pasa de una preponderante sensibilización de las manos, encargadas de "agarrar" agua para producir el desplazamiento en el medio acuático a una sobrecarga sensorial y motriz sobre los pies, antes olvidados atrás de todo, ahora base de la carrera. Talón, metatarso, puntas del pie, se encargan de sostener y proporcionar el apoyo en el que se imprime la fuerza para la carrera. El atleta vivencia su cuerpo propio con cambios bruscos en relación con el espacio vivido, su orientación y ejes, en relación a su fuerza motriz y su desplazamiento, en relación a las partes de su cuerpo que protagonizan apoyos y desplazamientos. Una vez que el atleta toma su casco y se lo coloca, así como también sus zapatos de ciclismo, toma su bicicleta, corre a su lado hasta salir del parque cerrado y la monta comenzando la segunda etapa de la carrera.

\section{Segunda etapa: el ciclismo}

En esta etapa se pone en juego de manera destacada la relación entre el cuerpo y la bicicleta, lo que modifica nuevamente los ejes, la orientación, el modo de desplazamiento y la espacialidad del cuerpo propio. Merleau-Ponty afirma que "no es nunca nuestro cuerpo objetivo lo que movemos, sino nuestro cuerpo fenomenal" (1985, p. 123), también considerado como cuerpo vivido o cuerpo habitual. Pero ¿qué implicaciones tiene esta afirmación? Nuestro cuerpo objetivo tiene límites más o menos precisos, la piel marca el límite exterior de nuestro cuerpo, la parte más externa de lo propio. Y el contacto con el medio acuático, como señalé anteriormente, pone de manifiesto este límite. Sin embargo, afirma el autor francés que no es ese cuerpo objetivo el que movemos y, por lo tanto, los límites del mismo no son tan claros. 
Continúa más adelante Merleau-Ponty: "La habitud expresa el poder que tenemos de dilatar nuestro serdel-mundo, o de cambiar la existencia anexándonos nuevos instrumentos" (1985, p. 161). Este anexo vendría a realizarse sobre el esquema corpóreo y el arco intencional, pues cuando nos habituamos a manejar un auto, a usar un bastón o un sombrero, nos instalamos en dichos objetos, e inversamente los hacemos participar de la voluminosidad del propio cuerpo (p. 161). De esta manera, el espacio objetivo del auto pasa a formar parte del espacio vivido del conductor, quien conduce sabe si el auto pasa o no pasa por un espacio determinado, no mide objetivamente las distancias, así como el cuerpo sin prótesis pasa por una puerta sin medir el ancho de sus hombros. Aprender a andar en bicicleta implica un saber-cómo, un saber del cuerpo, entregado al esfuerzo corporal e intraducible a un contenido proposicional objetivo. Ese aprendizaje, en determinados niveles de experticia, incorpora a la bicicleta al espacio vivido. El cuerpo fenomenal de un ciclista, al andar en bicicleta, no se limita a la piel o la ropa, se extiende al cuadro, a las ruedas, a la forma, a las palancas de la bicicleta, al casco. El ciclista no hace cálculos objetivos, matemáticos, algorítmicos, para saber si puede doblar o no a determinada velocidad en una curva, si pasa o no pasa entre otras dos bicicletas. Doblar no es girar una determinada cantidad de grados, sino que implica una toma de postura global del cuerpo y de la bicicleta en relación con el medio, cierta inclinación del torso, cierta contracción abdominal, cierto pedaleo o posición estática de los pedales, cierta tensión de las manos sobre la forma y cierta predisposición de los dedos sobre la palanca de frenos. Esa habitud o habitualidad a medida que se afianza requiere cada vez menos esfuerzo para desenvolverse en el mundo. Así, la noción de entrenamiento se vuelve imprescindible para dar cuenta del aprendizaje de hábitos y de la relación del hombre con la máquina, para conformar un cuerpo fenomenal disponible y apto para desenvolverse y sobrellevar contratiempos y problemas. El tacto del ciclista no se limita en las yemas de los dedos, sino que siente los accidentes del asfalto en el rozamiento de la cubierta, percibe de distinta manera ese mismo asfalto según la presión que tengan las cubiertas, percibe el esfuerzo que realiza sobre las palancas. Los hábitos perceptivos y motores son solidarios en cuanto se puedan desarrollar en detalle.
La existencia del ciclista se prolonga en la bicicleta. La experiencia del cuerpo vivido del ciclista, así como la del momento de nadar, puede necesitar fundamentalmente la presencia de las manos, la respiración y, ante todo, la vivaz presencia de las piernas, las cuales son protagonistas del movimiento de rotación que posibilita que la bicicleta marche. Así ocurre desde que comienza hasta que termina la segunda etapa de la competencia, cuando el ciclista completa el circuito delimitado y vuelve al parque cerrado para comenzar la segunda transición.

\section{Segunda transición: del ciclismo al pedestrismo}

Una vez en el parque cerrado, el atleta desmonta la bicicleta, y vuelve, luego de haber cargado de esfuerzo sus piernas, a correr para dejar la bicicleta en su sitio determinado previamente. Las piernas se sienten ocupando un espacio mayor, se sienten más pesadas, el esfuerzo provoca cambios en el cuerpo tal como es experimentado. El atleta deja la bicicleta, el casco y se cambia su calzado, preparándose para transitar la tercera y última etapa de la competencia: el pedestrismo.

\section{Etapa final: el pedestrismo}

Al dejar la bicicleta y sus accesorios pertinentes en el parque cerrado comienza la última etapa de la competencia: la carrera o pedestrismo. En esta instancia, si bien no hay un predominio del sentido del tacto tan marcado como en la natación, este es mayor acá que en la etapa de ciclismo, donde predomina el sentido visual. Aquí se combinan todos los sentidos, pues el oído y el olfato estaban prácticamente anulados en el agua y habitualmente el viento disminuye la claridad auditiva y olfativa en el ciclismo. En el pedestrismo se vuelve a tener presencia de los cinco sentidos y sus sinestesias. La exterocepción, la propiocepción y la interocepción comienzan a reposicionarse constantemente en la experiencia del corredor, pues, si todo va bien, si fluye la carrera, el deportista tiene una experiencia perceptiva del entorno, con la mirada al frente vislumbra su dirección, su horizonte, sin ocuparse de mucho más que de sus pisadas y su braceo al correr; pero si aparece alguna molestia, algún dolor, el hígado o el estómago, la interocepción se torna protagonista, y la existencia antes dilatada y lanzada al mundo comienza a concentrarse en el cuerpo propio, a cerrarse, a focalizarse. El hígado dolorido 
gana espacio, se hace más grande, oculta los gritos de aliento de los espectadores y la alegría de saber que la línea de llegada está cerca. Diversas estrategias aplican los atletas ante estas situaciones. La primera y más fácil, pero quizás la menos frecuente, es dejar el esfuerzo, soltar, aflojar, caminar. Aquí tenemos un cruce con la ética que solamente dejaremos sugerido. Otra opción es trasladar la atención a la memoración presente de la llegada, o al recuerdo de otra llegada ya pasada felizmente, $\mathrm{o}$ a la imaginación de la cercana llegada; para distraerse, para motivarse, para intentar opacar el grito doliente del cuerpo. Una tercera opción es la que lleva la atención al propio cuerpo, propiocepción e interocepción, se busca tomar preciso conocimiento de las distintas posturas, de los procesos invisibles e inefables que ocurren en nuestro cuerpo. A mayor entrenamiento y mayor calidad de los mismos, mayor cantidad de herramientas tendrá a disposición el atleta para poder continuar con su mejor rendimiento. Una vez más percepción y motricidad se encuentran atadas, correlacionadas. Temas como el dolor, el esfuerzo, la relajación, son pasibles de una larga y detallada descripción fenomenológica y son temas que actualmente se tratan y se debaten. Estos tópicos que forman parte de la vida cotidiana, en el mundo deportivo tienen un papel central, lo que nos permite un acceso más claro a dichos fenómenos.

Por último, quisiera agregar la importancia de la noción de ritmo en las tres disciplinas, pero especialmente en esta última etapa. Graciela Ralón de Walton, en su artículo "El suelo de la naturaleza como lugar de arraigo en la cultura" (2008), trae el concepto de estética fisiológica, acuñado por Waldenfels. Esta estética fisiológica es una estética en un sentido amplio, ligando ritmos, cadencias, repeticiones en una zona de cruce entre la naturaleza y la cultura (Ralón, 2008, p. 214). Este entrecruzamiento se da en la vida personal de cada existente, por lo que cada atleta expresará sus propios ritmos, con su propio estilo (otro concepto que nos quedará por abordar en futuras investigaciones). Los ritmos articulan el movimiento, y con él, el sentido. Parte de estos movimientos son los movimientos respiratorios, las cadencias del braceo, la cadencia y el paso, la rotación. Lo corredores afinan y pulen su estilo mediante el entrenamiento, de la motricidad y de la percepción. El correr expresa el ritmo de la existencia del corre- dor, muestra sus ritmos vitales, su estética fisiológica propia, con sus esfuerzos, su fluidez, sus notas distintivas, su modo de andar, su modo de cargar con el propio peso, su manera de ser en el mundo.

De esta manera, hemos realizado un trayecto que recorrió las tres etapas del deporte triatlón, con sus dos transiciones, describiendo algunos aspectos fenomenológicos relevantes de cada etapa aplicando algunos conceptos merleaupontianos, tales como cuerpo propio, espacio vivido, esquema corporal, arco intencional y la incorporación de nuevas habilidades al mismo. La tarea por realizar es ardua y vasta, pero prometedora, tanto para el campo fenomenológico como para la filosofía del deporte, ya que nos permite evaluar la adecuación o inadecuación de distintos conceptos fenomenológicos a los fenómenos deportivos por un lado, y porque nos permite tener mayor cantidad de herramientas conceptuales para seguir pensando el mundo del deporte a partir de la reflexión filosófica. En esta última línea, se presenta el último punto de este artículo, en el que a partir de las descripciones fenomenológicas propuestas se reflexiona sobre la flexibilidad del cuerpo humano para adaptarse al medio y las distintas transformaciones que tienen lugar en el triatlón.

\section{TRANSFORMACIONES: LOS LÍMITES DEL CUERPO PROPIO Y EL RITMO DE LA EXISTENCIA}

En este punto, cabe señalar que los conceptos que hemos utilizado para la descripción fenomenológica del triatlón han sido brevemente explicitados y que sin dudas queda mucho trabajo por hacer. El hecho de que no existan investigaciones previas en esta línea me hizo tomar la decisión de presentar algunos conceptos fenomenológicos relevantes y aplicarlos en aspectos nodales de la práctica del triatlón. De la misma manera, las conclusiones que podemos extraer de estas descripciones y su análisis, solamente nos va a proporcionar un eje de pensamiento que puede y debe ser desarrollado en futuras investigaciones. Michel Serres, en su obra Variaciones sobre el cuerpo (2011), señala que en el alpinismo, a partir del análisis de la relación del hombre con la montaña, se pueden extraer determinados aspectos filosóficos relevantes respecto al lugar que ocupa el hombre en el mundo. Su verticalidad, su bipedestrismo, 
son marcas señaladas de la especie humana, con su cuerpo que se mantiene en pie y camina. Pero no solo se pone en juego la animalidad del hombre, en el alpinismo, se pone en juego la cultura con el agarrar la piedra, y con el dejarla, las manos, que agarran y suelta, marcan el gesto que da comienzo a la cultura, según Serres (2011, pp. 44-45). Y en este enfrentamiento, acoplamiento, con la montaña el hombre se transforma, las plantas de los pies se hacen manos, el bípedo es cuadrúpedo, ya que sube, asciende y se despoja de lo que es para ser otra cosa. Posturas y posiciones inimaginadas adopta el hombre en los ascensos y en los descensos: dolor, esfuerzo y equilibrio que todo el tiempo se quiebran y buscan recomponerse.

De la misma manera, en el triatlón, la existencia muestra cierto devenir. En el agua, el principio, y tal como lo señala Gastón Bachelard en El agua y los sueños (2003), emerge la relación entre los fenómenos acuáticos y el origen de la vida, el útero materno. Estar envueltos por el medio acuático, y más aún si la temperatura del agua es agradable, podría referir a ese comienzo de la existencia. Fenomenológicamente marcamos la implicancia de este fenómeno con la constitución del cuerpo propio en la envoltura, el acogimiento, y en una predominancia del sentido del tacto y los datos propioceptivos.

Aprender a moverse, aprender a andar, comenzar a configurar un estilo propio de moverse, implica el desarrollo de un ritmo propio, aspecto que Merleau-Ponty marca como singular de cada existente. La primera transición marca los primeros pasos, y la relación con la máquina-útil, junto con los cambios que eso implica para el propio cuerpo y su espacialidad vivida. Señalamos el equilibrio como uno de los puntos destacados de la etapa del ciclismo. Merleau-Ponty señala en La estructura del comportamiento que, si bien los monos tienen una gran capacidad para equilibrar su cuerpo, tienen grandes dificultades para poner en equilibrio otros objetos. En nuestro fenómeno, se muestra que el ciclista pone en equilibrio su propio cuerpo en relación con la máquina, y ambos de acuerdo con los cambios dinámicos que le ofrece el medio. El pedestrismo viene marcado por el ritmo, el dolor, el esfuerzo y el cansancio que repercuten en el cuerpo vivido y en esa dialéctica desequilibrada en que el cuerpo se lanza hacia adelante, va despojándose de todo lo anterior, pues los esfuerzos y las vivencias de las etapas anteriores se encuentran sedimentados en el cuerpo, retenidos, "reprimidos" diría Merleau-Ponty. Cada corredor lleva su existencia como puede, con sus dolores, con sus cadencias, con sus posibilidades según su entrenamiento, sus pensamientos y sus emociones. Así, se entiende que los atletas habitualmente digan que el triatlón es una "forma de vida", entendemos que forma tiene que ver con el ritmo, la cadencia y las intensidades que se configuran en una estética fisiológica - en palabras de Waldenfels- y una manera de existir, un modo de ser en el mundo.

\section{REFERENCIAS}

Bachelard, G. (2003). El agua y los sueños. México: Fondo de Cultura Económica.

Laregina, A. (2013). Cuerpo propio y movimiento en el medio acuático: reflejos fenomenológicos. En Casetta, G. e Ibarra, A. (comps.), Representación en ciencia $y$ arte (vol. 4, pp. 665-675). Córdoba: Ed. Brujas-unc/ SECyTISBN

Leder, D. (1990). The absent body. Chicago: The University of Chicago Press.

Martínková, I. (2015). Phenomenology and Sport. En M. McNamee y W. Morgan (eds.), Routledge Handbook of Philosophy of sport (pp. 178-191). Londres-Nueva York: Routledge.

Merleau-Ponty, M. (1976). La estructura del comportamiento. Buenos Aires: Ed. Hachette.

Merleau-Ponty, M. (1985). Fenomenología de la percepción. Barcelona: Planeta Agostini.

Merleau-Ponty, M. (2003). El mundo de la percepción. Buenos Aires: Fondo de Cultura Económica.

O'Neill, J. (1974). The spectacle of the body. Journal of Philosophy of Sport, 1(1), 110-122.

Pérez, J. L. (2011). La filosofía del deporte: temas y debates. Dilemata, (5), 73-98.

Ralón de Walton, G. (2008). El suelo de naturaleza como lugar de arraigo en la cultura. En Investigaciones Fenomenológicas: Anuario de la Sociedad Española de Fenomenología (Serie Monográfica 1, pp. 207-772). Madrid: Universidad Estatal a Distancia (UNED), Sociedad Española de Fenomenología. 
Serrano de Haro, A. (1997). Fundamentos del análisis fenomenológico del cuerpo. En La posibilidad de la fenomenología (pp. 185-216). Madrid: Editorial Complutense.

Serrano de Haro, A. (2007). La precisión del cuerpo. Madrid: Ed. Trotta.

Serres, M. (2011). Variaciones sobre el cuerpo. Buenos Aires: Fondo de Cultura Económica.
Sheets-Johnstone, M. (2010). Body and Movement: Basic Dynamic Principles. En S. Gallagher y D. Schmicking (eds.), Handbook of Phenomenology and Cognitive Science (pp. 217-234). Dordrecht, New York, Heidelberg y London: Springer.

Torres, M. A. (2000). Triatlón deporte para todos. Barcelona: Editorial Paidotribo. 